\title{
Effects of Ion/Ion Proton Transfer Reactions on Conformation of Gas-Phase Cytochrome $c$ Ions
}

\author{
Qin Zhao, ${ }^{\text {a }}$ Gregg M. Schieffer, ${ }^{\text {a }}$ Matthew W. Soyk, ${ }^{\text {a,+ }}$ \\ Timothy J. Anderson, ${ }^{a}$ R. S. Houk, ${ }^{a, b}$ and Ethan R. Badman ${ }^{a, b, *}$ \\ a Department of Chemistry, Iowa State University, Ames, Iowa, USA \\ b Ames Laboratory U.S. Department of Energy, Iowa State University, Ames, Iowa, USA
}

Positive ions from cytochrome $c$ are studied in a 3-D ion trap/ion mobility (IM)/quadrupoletime-of-flight (TOF) instrument with three independent ion sources. The IM separation allows measurement of the cross section of the ions. Ion/ion reactions in the 3-D ion trap that remove protons cause the cytochrome $c$ ions to refold gently without other degradation of protein structure, i.e., fragmentation or loss of heme group or metal ion. The conformation(s) of the product ions generated by ion/ion reactions in a given charge state are similar regardless of whether the cytochrome $c$ ions are originally in +8 or +9 charge states. In the lower charge states $(+1$ to +5$)$ cytochrome $c$ ions made by the ion/ion reaction yield a single IM peak with cross section of $\sim 1110$ to $1180 \AA^{2}$, even if the original +8 ion started with multiple conformations. The conformation expands slightly when the charge state is reduced from +5 to +1 . For product ions in the +6 to +8 charge states, ions created from higher charge states $(+9$ to +16$)$ by ion/ion reaction produce more compact conformation(s) in somewhat higher abundances compared with those produced directly by the electrospray ionization (ESI) source. For ions in intermediate charge states that have a variety of resolvable conformers, the voltage used to inject the ions into the drift tube, and the voltage and duration of the pulse that extracts ions from the ion trap, can affect the observed abundances of various conformers. (J Am Soc Mass Spectrom 2010, 21, 1208-1217) (C) 2010 Published by Elsevier Inc. on behalf of American Society for Mass Spectrometry

$\mathrm{T}$ The determination of protein conformation is important in many biological applications. Of the various methods for these measurements, mass spectrometry (MS) has certain advantages such as speed and the need for only small amounts of sample. The variations of MS for study of protein conformation include ion mobility (IM) [1-4], H/D exchange [5-9], and native electron capture dissociation (NECD) [10, 11]. Of these methods, IM provides a direct way to examine the gas-phase conformation of the ions by probing the average cross-section of the protein ions via collisions with buffer gas [3,4]. Early IM research on protein folding and unfolding was done with an IMquadrupole instrument [1]. To study ions in lower charge states than those made directly from the electrospray ionization (ESI) source, a basic collision gas (e.g., acetophenone or 7-methyl-1,3,5-triazabicyclo 4,4,0] dec5-ene, MTBD) [12] was introduced into the source region. The neutral gas extracted protons from the

Address reprint requests to Dr. R. S. Houk, Department of Chemistry, Iowa State University, Ames IA 50011, USA. E-mail: rshouk@iastate.edu

* Current address: Hoffman-La Roche Inc., Nonclinical Safety, Nutley, NJ 07110 USA, E-mail: ethan.badman@roche.com

+ Current address: LECO, 3000 Lakeview Ave., St. Joseph, MI 49085. protein ion and created lower charge state ions through proton transfer reactions in the source. In these studies, the reactions took place only in the atmospheric pressure ion source interface region. Thus, control and variation of the reaction time and extent of reaction were difficult, and only certain reagent species were available.

Gas-phase ion/ion reactions provide another dimension for gas-phase bioanalysis by MS. To date, these reactions have been mainly used to simplify complex MS/MS spectra [13, 14] or generate fragments for structural assignment $[15,16]$ by methods like electrontransfer dissociation (ETD) [17-19].

Recent instrumentation improvements have greatly extended the type of structural information and number of possible experiments available in this area. The development of a 3-D trap-IM-time of flight (TOF) instrument allows time-dependent studies of gas-phase protein ions, including folding, unfolding and structural transitions [20-23]. A multi-stage IMS-MS instrument $[24,25]$ provides two important new functions. First, a protein ion with a specific structure can be selected by IMS, then activated and separated in the next drift region. Second, "state-to-state" structural 
transitions can be studied by structure selectionactivation cycles.

A three-source-ion trap-drift tube-q-TOF instrument was recently developed by our group to combine ion/ ion reactions with IM-TOF measurements [26, 27]. The present paper describes how proton transfer ion/ion reactions can be used to manipulate charge state and study the effect of ion/ion reactions on the conformations of cytochrome $c$ ions. This protein is chosen so the present results can be compared with those from extensive previous studies [10,22, 23, 28-36].

\section{Experimental}

The design and general operating conditions for the home-built three-source instrument are described in another paper [26]. Bovine heart cytochrome $c$ (SigmaAldrich, St. Louis, MO, USA) was used without further purification in all experiments. Usually this protein was dissolved at 20 to $30 \mu \mathrm{M}$ in water with $1 \%$ aqueous acetic acid. Water alone was used as solvent with $15 \mu \mathrm{M}$ protein for one set of results; this lower protein concentration suppressed cytochrome $c$ dimer ions, which were otherwise abundant from unbuffered water solutions. Another study used 50\% methanol/water with $1 \%$ acetic acid. These samples were introduced through one nano-ESI source in positive mode.

Positive cytochrome $c$ ions were stored in the trap first, then negative ions were added by switching the voltages on the ion optics. Negative ions from perfluoro1,3-dimethylcyclohexane ( $\mathrm{PDCH}, \mathrm{C}_{8} \mathrm{~F}_{16}$, Sigma-Aldrich, St. Louis, MO, USA) were used as the proton transfer reagent. Negative PDCH ions were created by atmospheric sampling glow discharge ionization (ASGDI) in a second source $[37,38]$. These $\mathrm{PDCH}$ negative ions were roughly $60 \%[\mathrm{M}-\mathrm{F}]^{-}$(i.e., $\mathrm{C}_{8} \mathrm{~F}_{15}{ }^{-}$) and the rest $[\mathrm{M}-$ $\left.\mathrm{CF}_{3}\right]^{-}[38,39]$.

Both positive cytochrome $c$ ions and negative $\mathrm{PDCH}$ ions were trapped together in the 3-D trap and allowed to react for times between 50 to $200 \mathrm{~ms}$, with the reaction time selected to optimize the yield of product ion in the desired charge states. Thus, the cytochrome $c$ ions spent $\sim 100$ to $300 \mathrm{~ms}$ in the trap. In this time regime, the mobility spectra and relative abundances of various cytochrome $c$ conformers were not strongly dependent on trapping time, as seen by Badman et al. $[22,23]$ and verified by us [27]. These time-dependent folding-unfolding effects were seen mainly for 9+ ions; most of the present work deals with lower charge states.

The product ions were then injected into the drift tube for IM separation, followed by $\mathrm{m} / \mathrm{z}$ analysis and detection by TOF-MS. The drift tube was $44.45 \mathrm{~cm}$ long and contained helium at 1.3 to 2 mbar. The default operating conditions for ion mobility measurement were as follows. The ion trap ejection pulse was zero to $-100 \mathrm{~V}$ for $3 \mu \mathrm{s}$, and the injection voltage on the drift tube entrance was $-50 \mathrm{~V}$. These conditions provided the best signal and minimized variation of IM spectra with duration of the injection pulse [26]. The injection voltage was varied for Figure 1, and $-50 \mathrm{~V}$ was used for Figure 6, for reasons discussed below.

The axial electric field in the drift tube was 12 to 13 $\mathrm{V} / \mathrm{cm}$. Cross sections were calculated from the mass

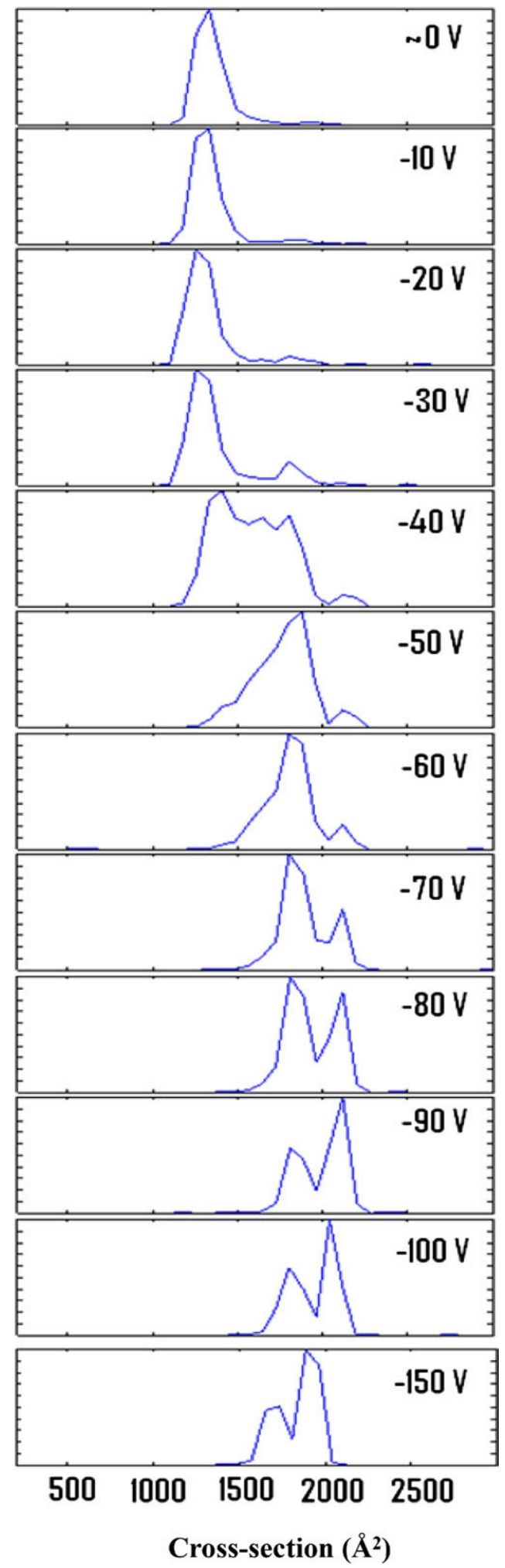

Figure 1. Cross-section distributions for cytochrome $c 8+$ ions at different injection voltage into the drift tube. 
resolved mobility spectra as described previously $[3,26]$. In particular, the drift time is calculated by measuring the total time between the ion trap exit pulse and the observation of ions at the TOF detector. The flight time between the exit of the drift tube and the TOF source region is calculated from the instrument dimensions, applied voltages and $\mathrm{m} / \mathrm{z}$ ratio and subtracted from the total time to give the actual time spent in the drift tube [26].

The number of measured points in the mobility spectra are determined by the time for the slowest ion to reach the TOF detector, which can be as long as $100 \mu \mathrm{s}$ for ions in low charge states. This limits the ability to resolve fine structure in the mobility spectra. The actual number of points in the mobility spectra are plotted in representative examples shown below. For charge states that yield single mobility peaks, cross section values are reproducible to $\sim 50 \AA^{2}$ from day to day; this uncertainty value is also affected by the number of points across the mobility peaks.

\section{Results and Discussion}

\section{Effect of Injection Voltage and Ion Trap Ejection Conditions on Conformations of Cytochrome $\mathrm{c}+8$ Ions}

In this device, there is a plume of gas leaving the drift tube ( $\sim 1 \mathrm{mbar})$ and moving toward the exit of the ion trap. Ions from the trap pass through this plume as they enter the drift tube. Clemmer's group [12] found that collisions with helium in this plume can heat and unfold protein ions, especially for proteins in intermediate charge states that have various conformations available. Their results mainly deal with the effect of the injection energy as controlled by the charge state and the voltage on the entrance to the drift tube. The effects observed are generally attributed to heating and unfolding in the initial collisions. The resulting mobility spectra can then be affected by cooling induced by subsequent collisions later in the drift tube.

Clemmer's group mainly reports results at moderate to high injection energy, i.e., lab energy of $385 \mathrm{eV}$ or 64 $\mathrm{V}$ for ubiquitin +6 to $38 \mathrm{~V}$ for ubiquitin +10 ions [12]. Our initial paper [26] reports similar effects for ubiquitin, including results at injection energies as low as 0 $\mathrm{eV}$, i.e., both ion trap and drift tube entrance at ground. As shown in Figure 1, at extremely low injection voltage, the cytochrome $c 8+$ ions are all in compact conformation(s), i.e., relatively low cross sections. As the injection voltage increases to $-30 \mathrm{~V}(240 \mathrm{eV}$ lab energy), some of the compact conformers start to unfold. As injection voltage increases above $-40 \mathrm{~V}(320$ $\mathrm{eV})$ to $-70 \mathrm{~V}(560 \mathrm{eV})$, the ions progress through partially folded to elongated structures, as described in the literature [22]. Roughly $30 \%$ of the $8+$ ions do not unfold completely even at the highest injection voltage used, $-150 \mathrm{~V}$, for a lab kinetic energy of $1200 \mathrm{eV}$. This is the value used for most of the results presented below.
In our previous work [26], conformation changes in ubiquitin $7+$ are described as the ejection pulse duration changes. Similar effects are observed with cytochrome $c 8+$ and $7+$. As with injection energy effects, these conformation changes are most extensive for those ions in intermediate charge states with various accessible conformations. If ions in these "intermediate" charge states are close to, but not inside, the entrance to the drift tube when the ion trap ejection pulse goes to zero, the ions can unfold. Such changes are also most extensive if the ion trap exit voltage is more negative than the drift tube entrance voltage. Ions in low or high charge states show less extensive folding changes with ejection pulse duration. Experimentally, longer ejection pulses improve the abundance of ions in low charge states, which is how the effect of ejection pulse duration was first found. The precise causes of this effect are being studied further.

For the results presented above, experimental conditions were used that minimize variations in conformation with injection pulse duration. In particular, changes in folding and conformation that depend on ejection pulse duration are minimal if the drift tube entrance voltage (usually $-150 \mathrm{~V}$ ) is kept more negative than the ion trap ejection pulse $(-100 \mathrm{~V})$.

\section{Effect of Charge Reduction Reaction on the Conformation of Cytochrome c Ions}

In $1 \%$ acetic acid/water solution, ESI produces cytochrome $c$ ions in two main charge states, $8+$ and 9+ (Figure 2) [1]. Although their IM drift times overlap, resolved mobility peaks for the +8 and +9 ions can be extracted by selecting the desired $\mathrm{m} / \mathrm{z}$ window in the 3D mass-mobility spectrum. Note that the $8+$ ion has two resolvable mobility peaks, which are generally attributed to different gas-phase conformations. The cross sections for these two peaks are $\sim 1710$ and $1900 \AA^{2}$ (Table 1); this difference represents the minimum resolvable difference in cross section seen so far with this particular device.

In the mass spectrum the main peaks from cytochrome $c$ are accompanied by less abundant satellite peaks at higher $\mathrm{m} / \mathrm{z}$ values. These latter peaks were originally thought to be adduct ions made inside the device, perhaps by addition of diffusion pump oil molecules to the protein ions while they traverse the drift tube [40]. However, these satellite peaks are also observed when the same solutions are analyzed with other mass spectrometers that do not have diffusion pumps, so they are attributed mainly to impurities in the cytochrome $c$ samples [41].

To study how ion/ion reactions affect the conformation of protein ions, either +8 or +9 ions are isolated in the 3-D ion trap first. The cross section plots are similar to those obtained when both +8 and +9 ions are stored and ejected into the drift tube together without $\mathrm{m} / \mathrm{z}$ isolation inside the ion trap (data not shown) [42]. If the 

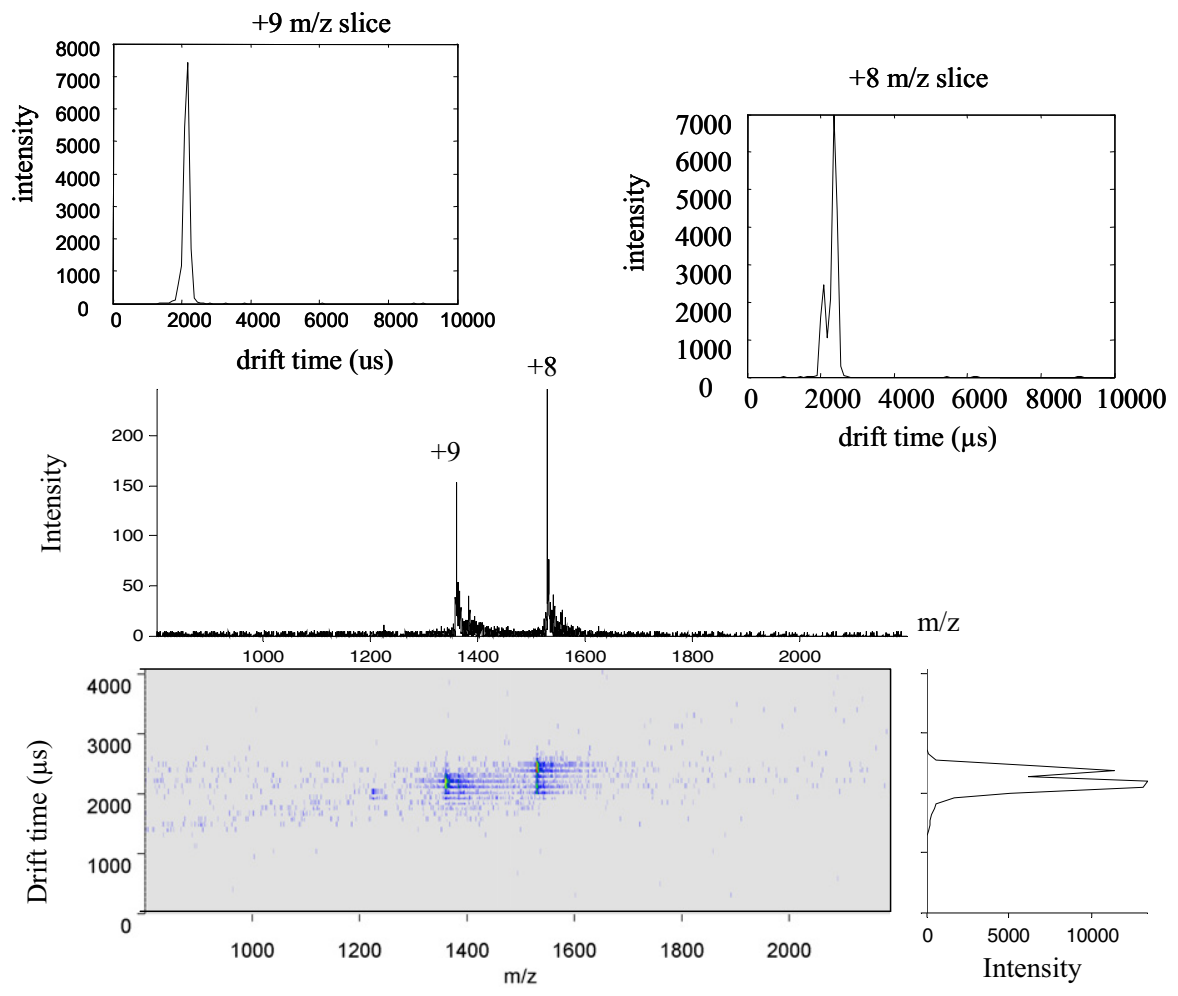

Figure 2. Nested 3-D IM-TOF mass spectrum of $30 \mu \mathrm{M}$ cytochrome $c$ in water with $1 \%$ acetic acid. The summed mass and ion mobility spectra are in the middle and at the lower right, respectively. The extracted IM spectra for cytochrome $c 8+$ and $9+$ ions are displayed at the top.

storage time in the trap is increased to at least $200 \mathrm{~ms}$, the mobility spectrum remains the same, so the ions do not heat and unfold appreciably in the trap (data not shown) [42].

$\mathrm{PDCH}$ negative ions are then injected into the ion trap from the ASGDI source and react with cytochrome

Table 1. Average cross-sections for cytochrome $c$ ions in different charge states $\left(\AA^{2}\right)$

\begin{tabular}{|c|c|c|c|c|}
\hline \multirow[b]{2}{*}{$\frac{\text { Charge }}{\text { State }}$} & \multicolumn{2}{|c|}{$\begin{array}{l}\text { Measured in } \\
\text { present work }\end{array}$} & \multicolumn{2}{|c|}{ Literature values } \\
\hline & $\begin{array}{c}\text { From } \\
9+\end{array}$ & $\begin{array}{c}\text { From } \\
8+\end{array}$ & $\begin{array}{c}\text { Shelimov } \\
{[45]}\end{array}$ & $\begin{array}{l}\text { Badman } \\
\text { [23] }\end{array}$ \\
\hline $9+$ & 1975 & & $\begin{array}{l}1964 \\
2215\end{array}$ & \\
\hline $8+$ & $\begin{array}{l}1468 \\
1715\end{array}$ & $\begin{array}{l}1709 \\
1900\end{array}$ & $\begin{array}{c}1250 \\
1702,1845 \\
2061\end{array}$ & $\begin{array}{r}1260 \\
1477,1709 \\
1854,2071\end{array}$ \\
\hline $7+$ & $\begin{array}{l}1493 \\
1708\end{array}$ & $\begin{array}{l}1488 \\
1704\end{array}$ & $\begin{array}{l}1247,1620 \\
1785,2007\end{array}$ & $\begin{array}{l}1257,1426,1629 \\
1792,2018\end{array}$ \\
\hline $6+$ & $\begin{array}{l}1179 \\
1364\end{array}$ & $\begin{array}{l}1085 \\
1359\end{array}$ & $\begin{array}{c}1244 \\
1393,1602\end{array}$ & $\begin{array}{r}1254 \\
1400,1611\end{array}$ \\
\hline $5+$ & 974 & 970 & 1196,1340 & $\begin{array}{l}1205 \\
1352\end{array}$ \\
\hline $\begin{array}{l}4+ \\
3+\end{array}$ & $\begin{array}{l}1015 \\
1073\end{array}$ & $\begin{array}{l}1069 \\
1071\end{array}$ & $\begin{array}{l}1153 \\
1139\end{array}$ & $\begin{array}{l}1160 \\
1150\end{array}$ \\
\hline $\begin{array}{l}2+ \\
1+\end{array}$ & $\begin{array}{l}1055 \\
1138\end{array}$ & $\begin{array}{l}1134 \\
1135\end{array}$ & & \\
\hline
\end{tabular}

$c$ ions to remove protons for the desired time. A typical spectrum after ion/ion reaction is shown in Figure 3. The resulting cross sections (Table 1) agree well with those reported [23]. The proton transfer reactions leave the protein ions in a range of charge states, which can be as low as $1+$ [26]. As expected, these reactions do not displace the covalently-bound heme group; proton transfer is gentle enough that the noncovalently-bound heme group in myoglobin remains after multiple proton transfer reactions $[43,44]$.

Cross-section values for cytochrome $c$ ions in each charge state, produced by reaction of isolated $8+$ or $9+$ ions with $\mathrm{PDCH}$ negative ions in the trap, are summarized in Table 1. The distributions of cross section observed for each charge state are indicated in Figure 4. These distribution plots indicate the relative abundances of the various conformers of cytochrome $c$ ions in the various charge states.

In general, the distributions of conformations for ions in a given charge state are similar whether the reactions started with either $8+$ or $9+$ ions. The wider mobility peaks seen for $7+$ and $6+$ ions after ion/ion reaction suggest that there may be several additional conformations that are not fully resolved. The ions in lower charge states $(5+$ to $1+)$ have only one mobility peak, even when the reactant ion was the $8+$ form with two mobility peaks originally. 

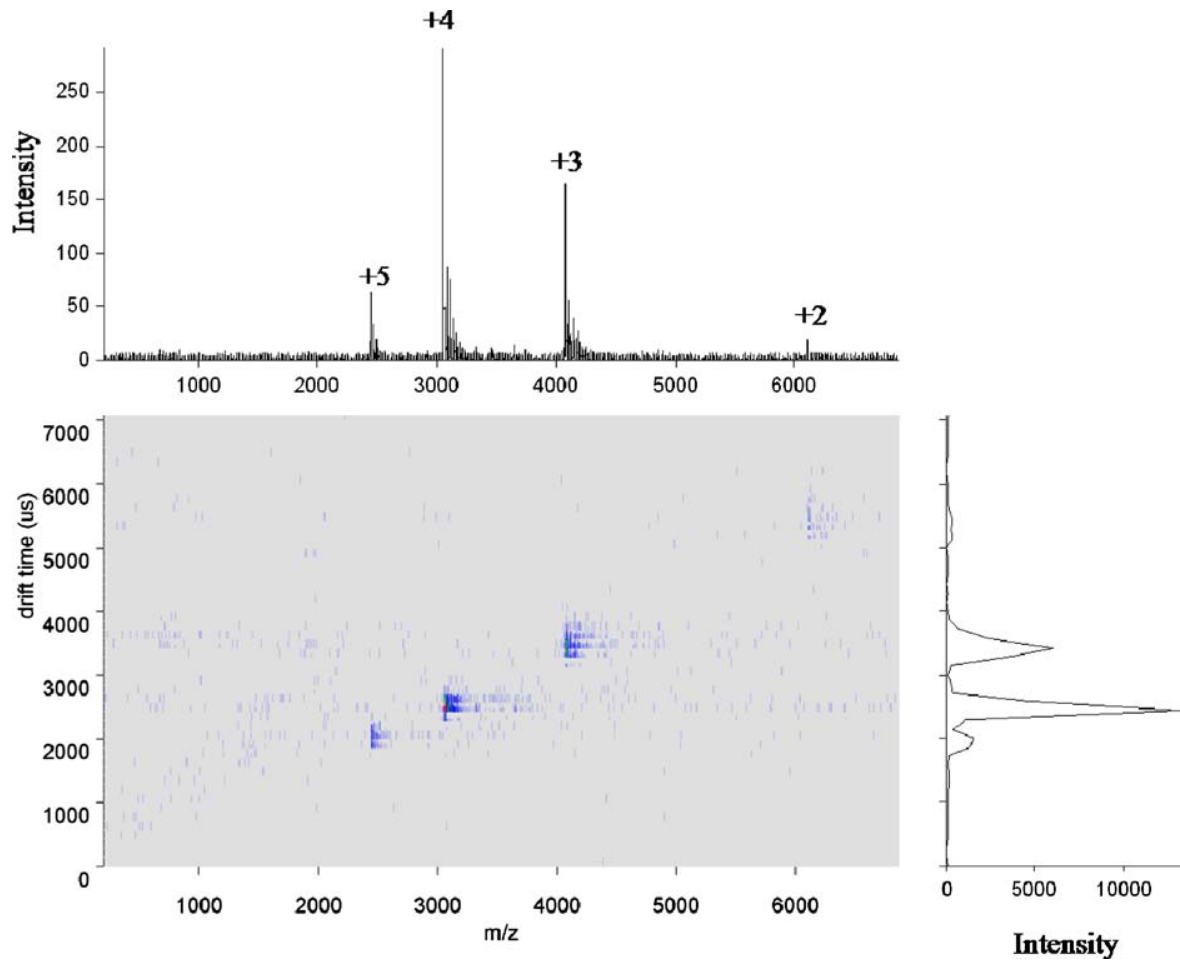

Figure 3. 3-D spectrum after isolated $8+$ cytochrome $c$ ions react with $\mathrm{PDCH}$ negative in the ion trap for $100 \mathrm{~ms}$.

Comparison of our cross section values with literature values (Table 1) can be summarized as follows:

(a) Our value for cytochrome $c 9+\left(1975 \AA^{2}\right)$ is close to the lowest literature value $\left(1964 \AA^{2}\right)$; we did not observe a conformer with cross section $2215 \AA^{2}$ for cytochrome $c 9+$.

(b) For charge states $5+$ and lower, our values are similar to but a bit smaller than those from the literature;
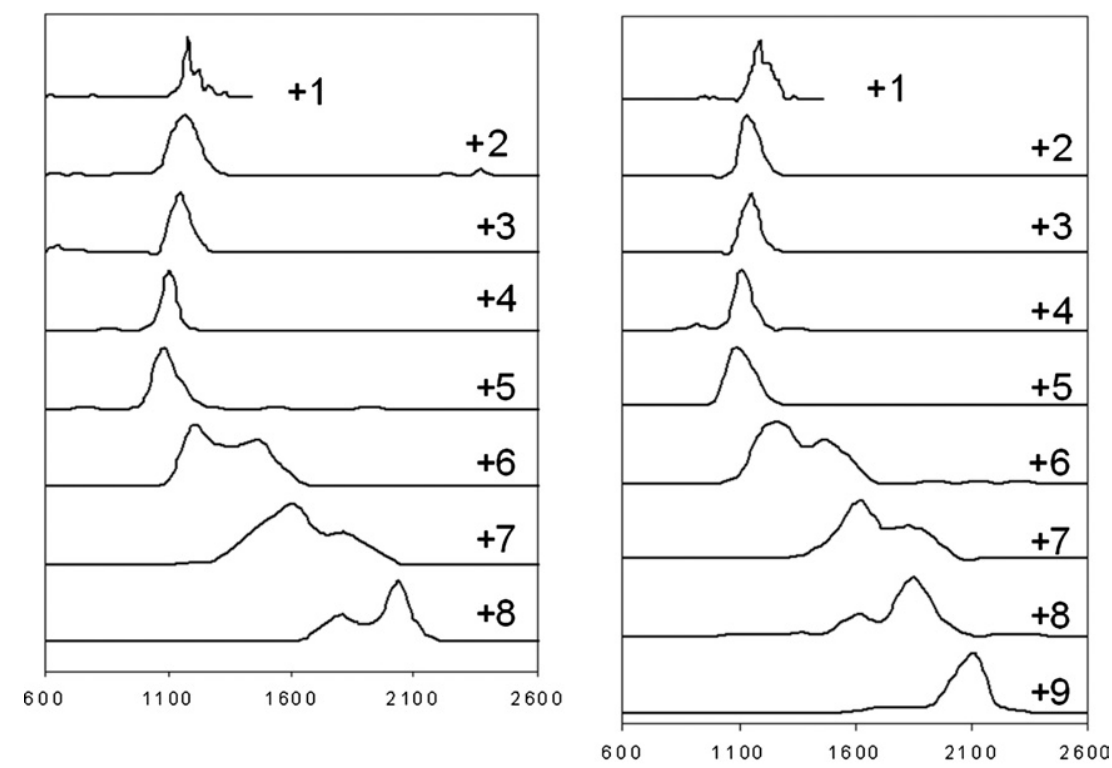

\section{Cross-section $\left(\AA^{2}\right)$}

Figure 4. Distribution of cross sections for cytochrome $c$ ions in each charge state. For the plots at the left, the cytochrome $c$ ions were initially in the $8+$ state; ion/ion reaction time was $50 \mathrm{~ms}$ to go to $4+$ and $110 \mathrm{~ms}$ to go to $1+$. The right plots are for charge reduction of $9+$ cytochrome $c$ ions for $40 \mathrm{~ms}$ to go to $4+$ and $80 \mathrm{~ms}$ to go to $1+$. 
(c) For charge states $6+$ to $8+$, we see fewer resolvable conformers, mainly because of our limited mobility resolution, see the Experimental section. For $8+$ and $7+$, we do not see the more folded conformers with cross sections $\sim 1250 \AA^{2}$.

The literature values noted in Table 1 from the work of Shelimov et al. [45] were measured for ions in lower and intermediate charge states that were made by proton transfer reaction with neutral gas during ion extraction. The other literature values listed from Badman et al. [23] were measured with an earlier IMSTOF-MS device similar to that used in the present work. More work is needed to determine if the observed differences are just instrumental effects or represent real differences in conformation(s) of ions depending on their origin.

It is tempting to assert that observation of just one mobility peak means the ions have only one conformation. The narrowest mobility peak seen for the $9+$ ion of cytochrome $c$ has a full width at half maximum (FWHM) of $\sim 180 \mu$ s (Figure 2). Calculations indicate the contribution to the fwhm of this peak from diffusion to be only $\sim 16 \mu \mathrm{s}$, much less than the peak width observed [46]. Under these experimental conditions, $\mathrm{Cs}_{7} \mathrm{I}_{6}{ }^{+}$ions or protonated reserpine ions (from ESI of CsI or reserpine in water) yield single mobility peaks $\sim 80 \mu \mathrm{s}$ fwhm, roughly half the width of the narrowest protein peaks. Thus, the "single" mobility peaks seen for cytochrome $c$ probably correspond to the juxtaposition of unresolved peaks from ions in several conformations of similar size, as shown by Clemmer et al. for ubiquitin [24]. For brevity, we use phrases like "one conformation" or "a single conformation" in the discussion below, with this caveat in mind.

These results show that the protein ions can fold to one or more compact conformations during the charge reduction reaction. This observation is explained as follows. When the protein ions pass into the vacuum system, the solvent molecules evaporate, counter ions are also lost, and the attraction between hydrophobic portions of the molecules diminishes. Thus, the highly charged protein ion opens rapidly to "partially unfolded" or "unfolded" conformations [11, 12, 23, 31, 32]. The ion/ion reaction in the ion trap then removes protons, whose charges keep the protein unfolded. Intramolecular charge repulsion becomes weaker as protons are removed, so the protein ions fold to more compact conformations, at least until very low charge states are achieved, as discussed below.

For cytochrome $c$ ions in the gas phase, ions with cross-sections $\sim 1050 \AA^{2}$ to $1350 \AA^{2}$ are generally assigned to "most compact" conformation(s) $[2,23]$. Thus, Figure 4 shows that the proton transfer reactions in the 3-D ion trap allow the original "unfolded" protein to pass gradually through several partially folded conformations and eventually assume compact conformations.

Close examination of Table 1 and the mobility plots in Figure 4 shows that the $5+$ ion has the smallest cross section, and cross sections for those ions in the "most compact" conformation increase slightly as charge state is reduced from $5+$ to $1+$. Apparently, as more protons are removed from the $5+$ ion, the cytochrome $c$ molecule expands by a small but measurable amount. This effect is also observed for ubiquitin in low charge states [26], but the size increase is not as extensive as for cytochrome $c$.

Possible reasons for this observed expansion at very low charge state are as follows. In low charge states, stabilization from ionic hydrogen bonds helps keep the protein ion folded. As the last few $\mathrm{H}^{+}$ions are removed, this stabilization is lost, and the protein ion expands somewhat [10, 11, 47]. A similar explanation is as follows. Starting with ions in high charge states, suppose the proton transfer reaction initially removes protons with the lowest gas-phase basicity, such as protons on lysine residues that are not shielded by other residues of the protein structure. After the ion has been converted to a low charge state, further reactions remove protons from the most basic residues (e.g., arginines) that are partially stabilized by noncovalent interactions with other residues. Thus, when these last protons are removed, some weak interactions on the protein surface are lost, the structure is then more flexible, and less compact.

\section{Conformation of Ions Made by Ion/Ion Reaction Compared with Those Produced Directly by ESI}

To generate ions in lower original charge states, cytochrome $c$ is sprayed from either of two solvents: (1) water without acetic acid or buffer, or (2) water buffered with ammonium acetate $(2 \mathrm{mM}, \mathrm{pH} \sim 7)$. These cytochrome $c$ ions are then measured without ion/ion reaction, and the results are compared with those obtained by charge reduction reactions of more highly charged ions from water/acetic acid solutions.

Figure 5 compares cross section distributions for ions in various charge states, prepared either by ion/ion reaction or by ESI directly. First, consider the results for the $7+$ and $6+$ charge states. For $7+$ ions made by ion/ion reaction, two broad mobility peaks are seen with about the same average cross section and relative abundances, regardless of whether the ions started as $9+$ or $8+$. For the $7+$ ions made directly by ESI, two mobility peaks are also seen, but the cross sections are different; the ions from water are more elongated, the ions from ammonium acetate buffer are more compact, compared with the $7+$ ions made by ion/ion reaction. A similar trend is seen for the $6+$ ions; here the elongated conformers are more abundant when the $6+$ ions are made by ion/ion reaction than directly by ESI.

For cytochrome $c 5+$, the ions made by ion/ion reaction have a single mobility peak at lower cross section than the ions from water directly but a little higher cross section than the $5+$ ions from ammonium acetate buffer. This single mobility peak gets narrower 


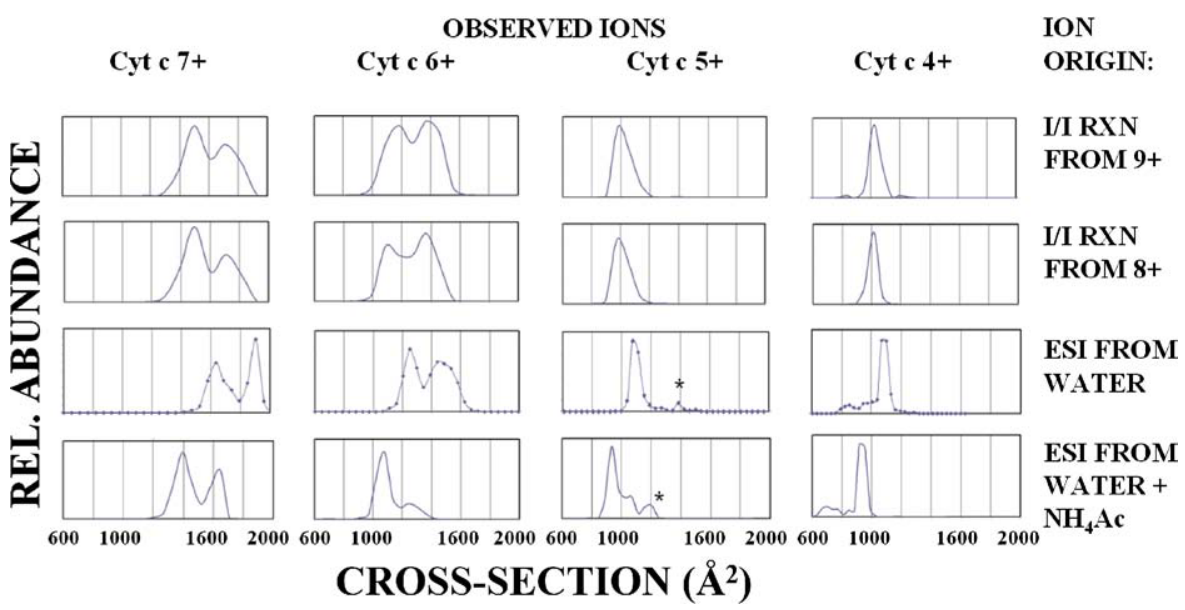

Figure 5. Distribution of cross sections for cytochrome $c$ ions in charge states $7+$ to $4+$. The ions are either made by ion/ion reaction from the $9+$ or $8+$ species in the ion trap, or observed directly by ESI from either water or aqueous ammonium acetate buffer. For the $5+$ ions, the asterisks denote an unusual "open" conformer seen only when these ions are made directly by ESI, not by ion/ion reaction.

for the $4+$ ions and approaches values $\sim 900 \AA^{2}$ for the ions from ammonium acetate, a value generally considered to be typical of compact forms. For the $5+$ and $4+$ ions made from water, the cross section of the main mobility peak is larger, $\sim 1100 \AA^{2}$.

There is also some interesting information from low-abundance mobility peaks. For the $4+$ ions made directly by ESI, from either water or ammonium acetate, small but measurable amounts of conformers at even smaller cross section are observed, in broad agreement with some previous measurements on cytochrome $c$, e.g., Figure 6 of reference [45]. The 5+ ions from water also have a small but measurable amount of larger conformer(s) at $\sim 1400 \AA^{2}$; these conformer(s) are not seen when the $5+$ ions are made either by ion/ion reaction of from ammonium acetate.

Thus, the mobility spectra can differ depending on the origin of the ions, particularly for intermediate charge states like those studied here that have various accessible conformers. This observation is relevant to several important issues. How much of the original solution structure is retained in the gas-phase ions observed in these and other ESI experiments [10, 11, 47]? If ions made by ESI in moderate or high charge states are trapped and reacted down to lower charge states, do they go back into conformations like those in the original solution? The apparatus and methodology described here could be used to help address these matters.

\section{"Stepwise" Proton Transfer Reactions}

For the ion/ion reactions described above, a single instrument step is used, and the fill and reaction times are varied to produce ions in particular charge states. For example, a reaction time of $\sim 50 \mathrm{~ms}$ converts the $8+$ ions mainly to $6+$ and $5+$, while making $2+$ and $1+$ ions requires reaction for $\sim 100 \mathrm{~ms}$.
These reactions can also be done in successive instrumental steps. The reaction can be stopped at a desired pair of charge states and then continued as desired. For example, positive ions in the $8+$ and $9+$ charge states are stored in the ion trap, and much smaller amounts of negative $\mathrm{PDCH}$ ions are injected, i.e., $\mathrm{PDCH}$ ions are added for fill times of only $\sim 10 \mathrm{~ms}$. The ion/ion reaction is allowed to proceed for $\sim 60 \mathrm{~ms}$; cytochrome c $7+$ and $8+$ ions result. Another $10 \mathrm{~ms}$ batch of PDCH ions are added, the reaction is continued for another 50 $\mathrm{ms}$, and the cytochrome $c$ ions $7+$ and $8+$ ions are converted to $6+$ and $7+$.

These sequential reactions can be continued to make ions in any pair of adjacent charge states down to $3+$ and 2+ [38]. For each instrumental step, careful control of the reaction time and reagent amount in the trap results in removal of only one charge. This method is an alternative to ion parking [48] to enhance the yield of ions in a particular charge state. The advantage is that it can avoid the collisional heating that could accompany the excitation step in ion parking, which may induce conformation changes in the protein ions. The mobility spectra of these ions made by this "stepwise" scheme are similar to those obtained from ions made "all at once" [39].

\section{Effect of Methanol on Charge State and Conformation of Ions Made by Ion/Ion Reaction}

Even if the ions generated by ESI are solvent-free, they may still retain some aspects of their conformation(s) in the original solution. For example, Breuker and coworkers $[31,32]$ report simulations that indicate that cytochrome $c$ unfolds partially soon after the solvent is removed, but that the gas-phase ions still have some of the structural components of the original folded protein. The final charge states of the ions, and presumably 
their eventual gas-phase conformations, can be manipulated to some extent by changing solvent conditions. For ubiquitin from water with $1 \%$ acetic acid, the more compact conformations observed are more abundant than for ubiquitin ions formed from 50/50 water/ methanol solution with $1 \%$ acetic acid [49].

To study the effect of initial solution conditions on the folding process induced by ion/ion reaction, cytochrome $c$ in 50/50 water/methanol solution with $1 \%$ acetic acid is sprayed. This solvent produces ions in higher initial charge states $(16+$ to $9+)$, as expected (Figure 6a) [39]. These highly charged ions yield single mobility peaks, again as expected if electrostatic repulsion keeps the ions unfolded. Ion/ion reaction with $\mathrm{PDCH}$ negative ions for various times converts these ions to charge states $8+$ to $2+$, with cross section distributions shown in Figure 6b. The same trend as in Figure 4 is found; the cytochrome $c$ ions fold to more compact conformations as charges are removed.

For these results, a lower injection voltage $(-50 \mathrm{~V})$ was used on the entrance to the drift tube to prevent fragmentation of the cytochrome $c$ ions in high charge states. Therefore, cross section plots for the $9+$ to $2+$ charge states obtained at $-50 \mathrm{~V}$ from $\mathrm{H}_{2} \mathrm{O}$ /acetic acid are also shown (Figure 6c). Comparison of Figure $6 b$ and $c$ show that, as charge state is reduced, the ions generally fold to similar conformations even when they start in very high charge states. For example, the $4+$ ions in Figure 6 b began as $9+$ to $16+$ but have a similar distribution of cross section as the $4+$ ions in Figure $6 c$, which began as $8+$ and $9+$. Open conformers are moderately more abundant for the $7+, 8+$, and $9+$ ions made from the more highly charged reactants (compare
Figure $6 \mathrm{~b}$ and $\mathrm{c})$. Also, the cross section distribution plots obtained for $8+, 9+$ ions and their products at an injection voltage of $-50 \mathrm{~V}$ (Figure 6c) are not greatly different from those obtained at $-150 \mathrm{~V}$ (Figure 4 ).

\section{Folding Transitions of Cytochrome c Ions Induced by Gas-Phase Ion/Ion Reaction}

From all the results discussed above, some general conclusions are:

(1) reduction of the charge state via ion/ion reaction can induce folding of the ions; (2) changes in folding of the elongated ions follow a similar pathway as that of the ions with partially folded structures; (3) ions in low charge states made by ion/ion reactions fold to similar conformations independent of their initial charge state; (4) for product ions in intermediate charge states $(5+$ to $8+)$, the distribution of conformers observed after ion/ ion reaction depends somewhat on the charge state of the reactant cytochrome $c$ ions; (5) the ions expand slightly as charge state is reduced from 5+ to $1+$; and (6) some partially unfolded conformers survive even when the ions are injected into the drift tube with high kinetic energies.

Starting with ions in high charge states that are unfolded in the trap, then reducing charge state via ion/ion reaction, is one way to induce and study folding changes in the ions. The observation that folding in the gas-phase yields conformers of similar abundance and cross section regardless of the initial conformation and charge state of the ions indicates these various ions fold via similar pathways. Dill and Chan
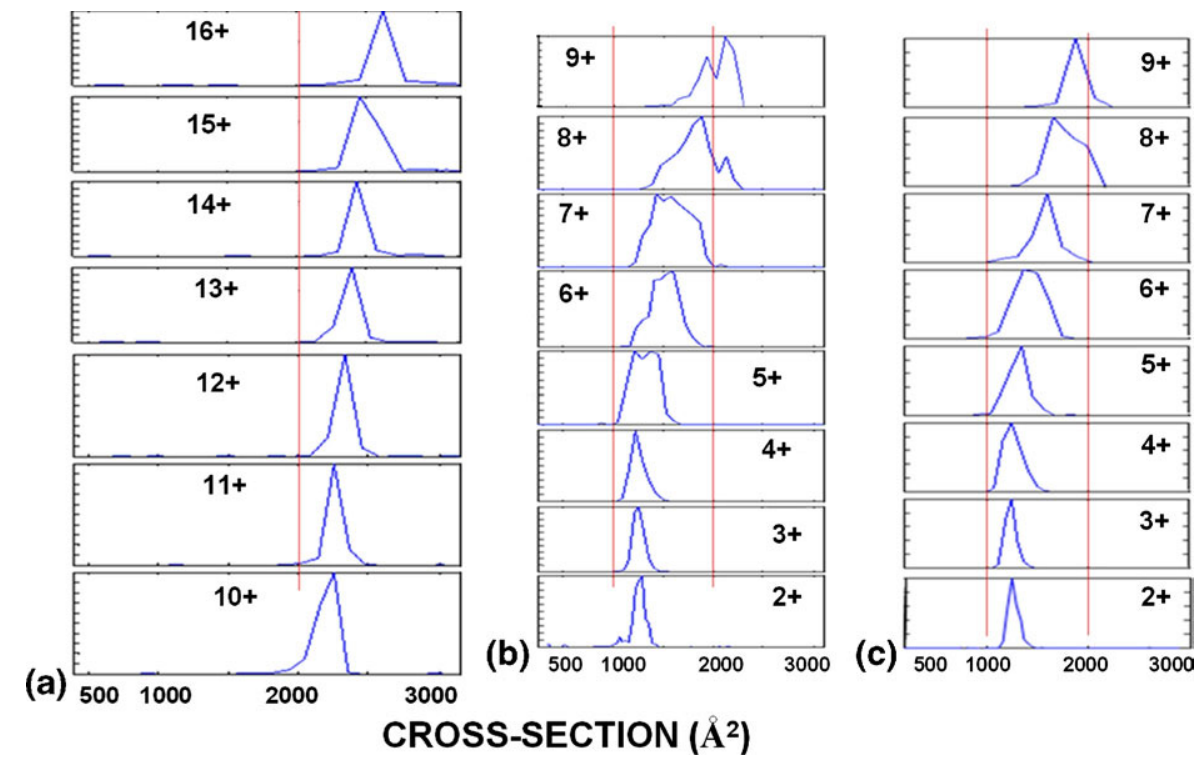

Figure 6. Distribution of cross section for cytochrome $c$ ions obtained with $-50 \mathrm{~V}$ on drift tube entrance. Red or gray lines have been added at 1000 and $2000 \AA^{2}$ for comparison: (a) cross sections of ions produced directly by ESI from $50 \% \mathrm{MeOH} / \mathrm{H}_{2} \mathrm{O}$ with $1 \%$ acetic acid; $(\mathbf{b})$ cross sections of ions produced by ion/ion reaction from cytochrome $c 9+$ to $16+$ from $50 \% \mathrm{MeOH} / \mathrm{H}_{2} \mathrm{O}$ with $1 \%$ acetic acid; (c) cross sections of cytochrome $c$ 8+ and $9+$ ions from $\mathrm{H}_{2} \mathrm{O}$ with $1 \%$ acetic acid and ion/ion reaction products for comparison. 
[50] describe protein folding mechanisms pictorially by "folding funnels" of several possible shapes; different conformers funnel into the native state finally. Our observations fit his "rugged energy landscape" model [50], which is a bumpy funnel with kinetic traps, energy barriers, and some narrow pathways through to the native state. Our research [26] and also that from Clemmer's lab [12] shows that the observed abundances of some conformers can be changed easily by increasing the injection energy, but some conformations remain very stable despite this heating process. The stable conformations represent the valleys in the bumpy folding funnel.

Our observations are also consistent with other depictions of protein folding, i.e., Englander's mechanism of folding through predetermined pathways with optional folding errors [34]. Specifically, Englander [51] finds a "misfolded" intermediate state in H/D exchange studies of cytochrome $c$ that could be analogous to a "bump" in the funnel in the viewpoint described by Dill [50].

\section{Conclusion}

Gas-phase ion-ion reactions combined with IM measurements provide a new way to study conformation changes in protein ions. Exothermic processes like these multiple proton transfer reactions might be expected to simply heat the ions and unfold them [52]. However the collisions with the bath gas in the 3-D trap cool the ions [53], so folded protein ions stay folded after proton transfer reaction. Thus, ion/ion reactions can be performed while the ions remain in, or perhaps re-fold into, biologically-interesting conformations.

The ion conformations formed via charge reduction reactions at relatively low-pressure inside the ion trap could also be compared with those formed from ion/ molecule reactions with neutral base gases in the ESI ion source, using reactions described by Zenobi [54, 55]. This experiment could probe the time frame over which ions liberated by ESI assume their final conformations, which is important to the key issue of whether the conformations of the ions produced by ESI resemble those in the solution [10,31, 32, 47].

In addition to the charge reduction reactions described here, this three-source ion trap-IM-TOFMS should facilitate other ion/ion reaction studies pertinent to bioanalysis. The identity and amount of reagent ion and the reaction time can be controlled over wide ranges. Other reactions such as ETD [17, 18], metal addition [56], and some sequential reactions (e.g., ETD followed by charge reduction to simplify assignment of the ETD fragments) should be possible.

The time progression of kinetic processes that change either the $\mathrm{m} / \mathrm{z}$ value or the conformation of the ions should be measurable, at least for processes that occur on time scales long compared with the duration of the measurement $(\sim 1 \mathrm{~s})$. The possibility of deliberately inducing unfolding and measuring its time duration with resolution of a few $\mu$ s [26] is intriguing. It should also be possible to induce folding processes with some time resolution by starting with unfolded ions in a high charge state and reducing the charge, then terminating the ion/ion reaction quickly, with subsequent measurement of conformation by IMS. These and other studies are underway in our laboratory.

\section{Acknowledgments}

The authors acknowledge funding for this work by a grant from the Vice Provost for Research, Iowa State University. Q.Z. and M.W.S. acknowledge the Conoco-Phillips Fellowship (Iowa State University, 2006-2007 and 2007-2008) for financial support. M.S. also acknowledges the GAANN Fellowship (Iowa State University, 2008) and the Velmer A. and Mary K. Fassel Fellowship (Iowa State University, 2006-2007). The authors thank Kathrin Breuker for suggesting one mechanism to explain the observed expansion of the ions at very low charge states. They also thank the three reviewers for helpful comments.

\section{References}

1. Hoaglund, C. S.; Valentine, S. J.; Sporleder, C. R.; Reilly, J. P.; Clemmer, D. E. Three-Dimensional Ion Mobility TOFMS Analysis of Electrosprayed Biomolecules. Anal. Chem. 1998, 70, 2236-2242.

2. Clemmer, D. E.; Hudgins, R. R.; Jarrold, M. F. Naked Protein Conformations-Cytochrome $c$ in the Gas-Phase. J. Am. Chem. Soc. 1995, 117, 10141-10142.

3. Clemmer, D. E.; Jarrold, M. F. Ion Mobility Measurements and Their Applications to Clusters and Biomolecules. J. Mass Spectrom. 1997, 32 577-592.

4. Bohrer, B. C.; Merenbloom, S. I.; Koeniger, S. L.; Hilderbrand, A. E.; Clemmer, D. E. Biomolecule Analysis by Ion Mobility Spectrometry. Annu. Rev. Anal. Chem. 2008, 1, 293-327.

5. Robinson, E. W.; Williams, E. R. Multidimensional Separations of Ubiquitin Conformers in the Gas Phase: Relating Ion Cross Sections to H/D Exchange Measurements. J. Am. Soc. Mass Spectrom. 2005, 16, 1427-1437.

6. Wood, T. D.; Chorush, R. A.; Wampler, F. M.; Little, D. P.; O'Connor P. B.; McLafferty, F. W. Gas-Phase Folding and Unfolding of Cytochrome $c$ Cations. Proc. Natl. Acad. Sci. U.S.A. 1995, 92, 2451-2454.

7. Freitas, M. A.; Hendrickson, C. L.; Emmett, M. R.; Marshall, A. G. Gas-Phase Bovine Ubiquitin Cation Conformations Resolved by GasPhase Hydrogen/Deuterium Exchange Rate and Extent. Int. J. Mass Spectrom. 1999, 187, 565-575.

8. Cassady, C. J.; Carr, S. R. Elucidation of Isomeric Structures for Ubiquitin $[\mathrm{M}+12 \mathrm{H}](12+)$ Ions Produced by Electrospray Ionization Mass Spectrometry. J. Mass Spectrom. 1996, 31, 247-254.

9. Freitas, M. A.; Hendrickson, C. L.; Marshall, A. G. Correlation Between Solution and Gas-Phase Protein Conformation: H/D Exchange, IRMPD, and ESI FT-ICR MS. Proc. SPIE Int. Soc. Opt. Eng. 2000, 3926, 61-68.

10. Breuker, K.; McLafferty, F. W. The Thermal Unfolding of Native Cytochrome $c$ in the Transition from Solution to Gas Phase Probed by Native Electron Capture Dissociation. Angew. Chem. Int. Edit. 2005, 44, 4911-4914.

11. Breuker, K.; McLafferty, F. W. Native Electron Capture Dissociation for the Structural Characterization of Noncovalent Interactions in Native Cytochrome. c. Angew. Chem. Int. Edit. 2003, 42, 4900-4904.

12. Valentine, S. J.; Counterman, A. E.; Clemmer, D. E. ConformerDependent Proton-Transfer Reactions of Ubiquitin Ions. J. Am. Soc. Mass Spectrom. 1997, 8, 954-961.

13. Stephenson, J. L.; McLuckey, S. A.; Reid, G. E.; Wells, J. M.; Bundy, J. L. Ion/Ion Chemistry as a Top-Down Approach for Protein Analysis. Curr. Opin. Biotechnol. 2002, 13, 57-64.

14. Stephenson, J. L.; McLuckey, S. A. Simplification of Product Ion Spectra Derived from Multiply Charged Parent Ions Via Ion/Ion Chemistry. Anal. Chem. 1998, 70, 3533-3544.

15. Breuker, K.; Oh, H. B.; Lin, C.; Carpenter, B. K.; McLafferty, F. W. Nonergodic and Conformational Control of the Electron Capture Dissociation of Protein Cations. Proc. Natl. Acad. Sci. U.S.A. 2004, 101 14011-14016.

16. Ge, Y.; Lawhorn, B. G.; ElNaggar, M.; Strauss, E.; Park, J. H.; Begley, T. P.; McLafferty, F. W. Top Down Characterization of Larger Proteins (45 KDa) by Electron Capture Dissociation Mass Spectrometry. J. Am. Chem. Soc. 2002, 124, 672-678.

17. Syka, J. E. P.; Coon, J. J.; Schroeder, M. J.; Shabanowitz, J.; Hunt, D. F. Peptide and Protein Sequence Analysis by Electron Transfer Dissociation Mass Spectrometry. Proc. Natl. Acad. Sci. U.S.A. 2004, 101, $9528-$ 9533. 
18. Pitteri, S. J.; Chrisman, P. A.; Hogan, J. M.; McLuckey, S. A. Electron Transfer Ion/Ion Reactions in a Three-Dimensional Quadrupole Ion Trap: Reactions of Doubly and Triply Protonated Peptides with $\mathrm{SO}^{-}$. Anal. Chem. 2005, 77, 1831-1839.

19. Chrisman, P. A.; Pitteri, S. J.; Hogan, J. M.; McLuckey, S. A. SO2Electron Transfer Ion/Ion Reactions with Disulfide Linked Polypeptide Ions. J. Am. Soc. Mass Spectrom. 2005, 16, 1020-1030.

20. Badman, E. R.; Hoaglund-Hyzer, C. S.; Clemmer, D. E. Dissociation of Different Conformations of Ubiquitin Ions. J. Am. Soc. Mass Spectrom. 2002, 13, 719-723.

21. Myung, S.; Badman, E. R.; Lee, Y. J.; Clemmer, D. E. Structural Transitions of Electrosprayed Ubiquitin Ions Stored in an Ion Trap over Similar to $10 \mathrm{~ms}$ to $30 \mathrm{~s}$. J. Phys. Chem. A 2002, 106, 9976-9982.

22. Badman, E. R.; Myung, S.; Clemmer, D. E. Evidence for Unfolding and Refolding of Gas-Phase Cytochrome $c$ Ions in a Paul Trap. J. Am. Soc. Mass Spectrom. 2005, 16, 1493-1497.

23. Badman, E. R.; Hoaglund-Hyzer, C. S.; Clemmer, D. E. Monitoring Structural Changes of Proteins in an Ion Trap over $\sim 10$ to $200 \mathrm{~ms}$ : Unfolding Transitions in Cytochrome c Ions. Anal. Chem. 2001, 73, 6000-6007.

24. Koeniger, S. L.; Merenbloom, S. I.; Clemmer, D. E. Evidence for Many Resolvable Structures within Conformation Types of Electrosprayed Ubiquitin Ions. J. Phys. Chem. B 2006, 110, 7017-7021.

25. Koeniger, S. L.; Merenbloom, S. I.; Sevugarajan, S.; Clemmer, D. E. Transfer of Structural Elements from Compact to Extended States in Unsolvated Ubiquitin. J. Am. Chem. Soc. 2006, 128, 11713-11719.

26. Zhao, Q.: Soyk, M. W.; Schieffer, G. M.; Fuhrer, K.; Gonin, M.; Houk, R. S.; Badman, E. R. An Ion Trap-Ion Mobility-Time of Flight Mass Spectrometer with Three Ion Sources for Ion/Ion Reactions. J. Am. Soc. Mass Spectrom. 2009, 20, 1549-1561.

27. Badman, E. R.; Schieffer, G. M.; Soyk, M.; Zhao, Q.; Anderson, T. J. An ESI-Ion Trap-Ion Mobility-q-TOF to Study Ion-Ion Reactions of Intact Biopolymers. Proceedings of the 53rd ASMS Conference on Mass Spectrometry and Allied Topics; San Antonio, TX, June, 2005.

28. Valentine, S. J.; Clemmer, D. E. H/D Exchange Levels of ShapeResolved Cytochrome $c$ Conformers in the Gas Phase. J. Am. Chem. Soc. 1997, 119, 3558-3566.

29. Englander, S. W.; Sosnick, T. R.; Mayne, L. C.; Shtilerman, M.; Qi, P. X.; Bai, Y. W. Fast and Slow Folding in Cytochrome c. Acc. Chem. Res. 1998, 31, 737-744.

30. McLafferty, F. W.; Guan, Z. Q.; Haupts, U.; Wood, T. D.; Kelleher, N. L. Gaseous Conformational Structures of Cytochrome c. J. Am. Chem. Soc. 1998, 120, 4732-4740.

31. Steinberg, M. Z.; Breuker, K.; Elber, R.; Gerber, R. B. The Dynamics of Water Evaporation from Partially Solvated Cytochrome $c$ in the Gas Phase. Phys. Chem. Chem. Phys. 2007, 9, 4690-4697.

32. Steinberg, M. Z.; Elber, R.; McLafferty, F. W.; Gerber, R. B.; Breuker, K. Early Structural Evolution of Native Cytochrome $c$ after Solvent Removal. Chem. Biochem. 2008, 9, 2417-2423.

33. Hoang, L.; Maity, H.; Krishna, M. M. G.; Lin, Y.; Englander, S. W. Folding Units Govern the Cytochrome $c$ Alkaline Transition. J. Mol. Biol. 2003, 331, 37-43.

34. Krishna, M. M. G.; Englander, S. W. A Unified Mechanism for Protein Folding: Predetermined Pathways with Optional Errors. Protein Sci. 2007, 16, 449-464

35. Krishna, M. M. G.; Lin, Y.; Rumbley, J. N.; Englander, S. W. Cooperative Omega Loops in Cytochrome $c$ : Role in Folding and Function. J. Mol. Biol. 2003, 331, 29-36.

36. Maity, H.; Rumbley, J. N.; Englander, S. W. Functional Role of a Protein Foldon-an Omega-Loop Foldon Controls the Alkaline Transition in Ferricytochrome c. Proteins Struct. Funct. Bioinform. 2006, 63, 349-355.
37. McLuckey, S. A.; Glish, G. L.; Asano, K. G.; Grant, B. C. Atmospheric Sampling Glow-Discharge Ionization Source for the Determination of Trace Organic-Compounds in Ambient Air. Anal. Chem. 1988, 60, 2220-2227.

38. Soyk, M. W.; Zhao, Q.; Houk, R. S.; Badman, E. R. A Linear Ion Trap Mass Spectrometer with Versatile Control and Data Acquisition for Ion/Ion Reactions. J. Am. Soc. Mass Spectrom. 2008, 19, 1821-1831.

39. Zhao, Q. Development of Ion Mobility Mass Spectrometry Coupled with Ion/Ion Reactions: Instrumentation and Applications for Protein Analysis. Iowa State University: Chap. 2 and 3.

40. Dugourd, P.; Hudgins, R. R.; Clemmer, D. E.; Jarrold, M. F. HighResolution Ion Mobility Measurements. Rev. Sci. Instrum. 1997, 68, 1122-1129.

41. McLuckey, S. A. Recent Developments in Ion/Ion Chemistry for Bioanalysis. Proceedings of the Federation of Analytical Chemistry and Spectroscopy Societies Conference; Memphis, TN, 2007.

42. Zhao, Q. Development of Ion Mobility Mass Spectrometry Coupled with Ion/Ion Reactions: Instrumentation and Applications for Protein Analysis. Iowa State University: Chap. 3

43. Stephenson, J. L.; VanBerkel, G. J.; McLuckey, S. A. Ion-Ion Proton Transfer Reactions of Bio-Ions Involving Noncovalent Interactions: Holomyoglobin. J. Am. Soc. Mass Spectrom. 1997, 8, 637-644.

44. Chrisman, P. A.; Newton, K. A.; Reid, G. E.; Wells, J. M.; McLuckey, S. A. Loss of Charged Versus Neutral Heme from Gaseous Holomyoglobin Ions. Rapid Commun. Mass Spectrom. 2001, 15, 2334-2340.

45. Shelimov, K. B.; Clemmer, D. E.; Hudgins, R. R.; Jarrold, M. F. Protein Structure in Vacuo: Gas-Phase Conformations of BPTI and Cytochrome c. J. Am. Chem. Soc. 1997, 119, 2240-2248.

46. Mason, E. A.; McDaniel, E. W. Transport Properties of Ions in Gases; John Wiley and Sons: New York, 1988, p. 86-91.

47. Breuker, K.; McLafferty, F. W. Stepwise Evolution of Protein Native Structure with Electrospray into the Gas Phase, 10(-12) to 10(2) s. Proc. Natl. Acad. Sci. U.S.A. 2008, 105, 18145-18152.

48. McLuckey, S. A.; Reid, G. E.; Wells, J. M. Ion Parking During Ion/Ion Reactions in Electrodynamic Ion Traps. Anal. Chem. 2002, 74, 336-346.

49. Li, J. W.; Taraszka, J. A.; Counterman, A. E.; Clemmer, D. E. Influence of Solvent Composition and Capillary Temperature on the Conformations of Electrosprayed Ions: Unfolding of Compact Ubiquitin Conformers from Pseudonative and Denatured Solutions. Int. J. Mass Spectrom 1999, 187, 37-47.

50. Dill, K. A.; Chan, H. S. From Levinthal to Pathways to Funnels. Nat. Struc. Biol. 1997, 4, 10-19.

51. Maity, H.; Maity, M.; Englander, S. W. How Cytochrome $c$ Folds, and Why Submolecular Foldon Units and Their Stepwise Sequential Stabilization. J. Mol. Biol. 2004, 343, 223-233.

52. Stephenson, J. L.; McLuckey, S. A. Ion/Ion Reactions in the Gas Phase: Proton Transfer Reactions Involving Multiply-Charged Proteins. J. Am. Chem. Soc. 1996, 118, 7390-7397.

53. Wells, J. M.; Chrisman, P. A.; McLuckey, S. A. Formation and Characterization of Protein-Protein Complexes in Vacuo. J. Am. Chem. Soc. 2003, 125, 7238-7249.

54. Touboul, D.; Jecklin, M. C.; Zenobi, R. Rapid and Precise Measurements of Gas-Phase Basicity of Peptides and Proteins at Atmospheric Pressure by Electrosonic Spray Ionization-Mass Spectrometry. J. Phys. Chem. B 2007, 111, 11629-11631.

55. Touboul, D.; Jecklin, M. C.; Zenobi, R. Investigation of Deprotonation Reactions on Globular and Denatured Proteins at Atmospheric Pressure by ESSI-MS. J. Am. Soc. Mass Spectrom. 2008, 19, 455-466.

56. Newton, K. A.; McLuckey, S. A. Gas-Phase Peptide/Protein Cationizing Agent Switching Via Ion/Ion Reactions. J. Am. Chem. Soc. 2003, 125, 12404-12405. 